[Aus der Abteilung für Augenkranke des Rud. Virch ow - Krankenkauses zu Berlin.]

\title{
Die Kombination von Punktion und Druckverband zur Behandlung der Netzhautablösung ${ }^{1}$ ).
}

\author{
Von
}

Dr. O. Fehr, dirigier. Arzt.

Nach einer Zeit unfruchtbarer Resignation, zu der die schlechten Erfahrungen mit den zahlreichen gegen die Netzhautablösung empfohlenen Behandlungsarten und pessimistische Ausserungen von Männern wie de Wecker'), Sattler ${ }^{3}$ ) und Uhthoff' gefuhrt haben, scheint seit kurzem das Interesse an der operativen Behandlung der Amotio retinae wieder reger zu werden. Neben Deutschmanus unermuidlicher Arbeit im Kampf gegen die Netzhautablösung ist das nicht zum wenigsten Birch-Hirschfeld zu danken. Seine Versuche mit Injektion von Flüssigkeit in den Glaskörper stehen zurzeit im Vordergrund des Interesses, Versuche, wie sie in ähnlicher Weise in früherer Zeit von Weber, Schoeler, Grossmann und vor allem von Deutschmann ${ }^{5}$ ) angestellt worden sind, die aber trotz aller theoretischer Begrïndung in der Praxis keine allgemeinere Anwendung haben finden können.

Schon 1909 hat Birch-Hirschfeld zusammen mit Inouyef) Vortrage.

1) Nach einem am 12. XII. 12 in der Berline* ophth. Ges. gehaltenem

2) de Wecker, Der heutige Stand der Therapie der Netzhautablösung. Die ophth. Klinik. 1899. 11.

3) Sattler, Über die Behandlung der Netzhantablösung. Dentsche med. Wochenschr. Nr. 1 u. 2. 1905.

4) Uhthoff, Über die Behandlung der Netzhantablösung. Samml. zwangl. Abb. aus d. Geb. d. Augenheilk. Bd. VI, 8. 1907.

5) Samtlich citiert von Wernicke. Klinische und experimentelle Beiträge zur operativen Behandlung der Netzhautablösung. Klin. Monatsbl. f, Augenheilk. Bd. I. 1906.

6) Birch-Hirsch feld u. Inouye, Experimentelle und histologische Untersuchungen über Netzhautablösung. v. Graefe's Arch. f. Ophth. Bd. LXX. S. 486.1909 . 
und 1911 allein $^{1}$ ) in diesem Archiv über das Ergebnis seiner ausgedehnten experimentell-histologischen Untersuchungen berichtet, welche die Feststellung der für die Genese der Amotio bedeutungsvollen anatomische Verhältnisse und des anatomischen Effektes der gebräuchlichen Operationen zur Aufgabe hatten. Er bestätigte die Ansicht Lebers und v. Hippels, „dass die Bildung und der elastische Zug präretinaler Membranen für die Entstehung und den Bestand einer Amotio von entscheidender Bedeutung sind, daneben aber Veränderungen, die sich im Gewebe der Netzhaut selbst abspielen, die zu deren Verkürzung, zu Faltenverklebung und Gliose, nicht selten zu Netzhautzerreissung führen". Birch-Hirschfeld fand, dass bei seinen Kaninchen, bei denen er durch Glaskörperabsaugung Netzhautablösung erzeugt hatte, die gewöhnlichen operativen Methoden nur dann erfolgreich waren, wenn die bezeichneten retinalen und präretinalen Veränderungen wenig entwickelt waren, und kommt zu dem Schluss, dass diejenige Operationsmethode den grössten Erfolg verspricht, welche nach Entleerung der subretinalen Flüssigkeit die Verwachsung der Netzhaut mit den Glaskörpersträngen und die verklebten Netzhautfalten zu lösen oder wenigstens zu entspamnen sucht. Letzteres glaubt er durch Injektion von Flüssigkeit in den Glaskörperraum zu erreichen; nur dadurch könne ein auf die ganze Oberfläche der Netzhaut wirkender Druck ausgeübt werden, der sie gegen die Lederhaut drückt. Von den Tierversuchen ging er zur Behandlung der menschlichen Amotio über. In einer dritten Arbeit $\left.{ }^{2}\right)$ beschreibt er das Verfahren, das er bisher an 19 Fällen exprobt hat: Nach Absaugung des subretinalen Exsudats mit der Pravaz-Spritze injizierte er unter Vermeidung von artfremdem Eiweiss, wie es Deutschmann benutzt, teils die abgesaugte subretinale Flüssigkeit, teils reine isotonische Kochsalzlösung, teils ein Gemisch von beiden. 8 mal gelang es ihm, eine $1 \mathrm{Jahr}$ beobachtete wesentliche Besserung mit Wiederanlegung der Netzhaut zu erzielen, 4 mal sah er eine geringe Besserung, 3 Fälle blieben unverändert, und in 4 Fällen kam es zum Fortschreiten des Leidens. Ein Resultat, das gewiss als recht gut bezeichnet werden muss.

Denselben Zweck wie Birch-Hirschfelds Versuche verfolgten

1) Birch-Hirschfeld, Experimentell-histologische Untersuchungen über Netzhautablösung und die Wirkung operativer Therapie. v. Graefe's Arch. f. Ophth. Bd. LXXIX, 2. S. 210. 1911.

2) Birch-Hirschfeld, Zur Therapie der Netzhautabhebung. v. Graefe's Arch. f. Ophth. Bd. LXXXII. S. 242. 1912. 
die von $\mathrm{Ohm}^{1}$ ), der nach der Punktion durch Injektion ron Luft in den Glaskörper die Netzhaut gegen ihre Unterlage anzupressen sucht. Am Menschen hat er die Methode, die sich am Katzenauge als unschädlich erwies, erst in 2 Fällen erprobt, von denen der eine geheilt wurde, der andere ungeheilt blieb. Krusius ${ }^{2}$ ) prüfte die $\mathrm{Ohm}$ sche Methode an Kaninchenaugen nach, in denen er Netzhautablösung durch Absaugen von Glaskörper erzeugt hatte. Die Luftinjektion geschah, ohne vorher die subretinale Flïssigkeit abzulassen. Letztere trat bei der Anpressung der Netzhaut an die Aderhaut neben der Kanüle durch einen feinen Netzhautriss in den Glaskörper. In den 8 Fällen blieb die Anlegung $7 \mathrm{mal}$ bis zu 3 Tagen bestehen, und nur einmal konnte dauernde Anlegung festgestellt werden. Krusius glaubt daher nicht, dass diese Versuche für die Praxis Bedeutung haben. Optimistischer äussert sich Rohmer ${ }^{3}$ ), der die Ohmsche Methode in 8 Fällen am Menschen angewandt hat. Birch-Hirschfeld, der, unabhängig von Ohm, auch die Luftinjektion in den Glaskörper in 2 Fällen von Netzhautablösung beim Menschen ohne Eifolg versuchte, hält diese Methode nicht für zweckmässig.

Derselbe Gedanke, der Deutschmann, Birch-Hirschfeld und Ohm bei ihren Versuchen leitete, nämlich, dass die vollständige Entleerung der subretinalen Ansammlung, sowie die Ausübung eines gleichmässigen Druckes auf die Netzhaut eine Bedingung für die Heilung der Netzhautablösung ist, bestimmt mich seit 5 Jahren, der Punktion der Netzhautablösung sofort einen energischen Druckverband folgen zu lassen. Ich glaube, dass durch den Druckrerband nach der Punktion ähnliches erreicht wird, wie durch die Injektionen in den Glaskörper, deren Ungefährlichkeit, auch wenn sie mit arteigenem Eiweiss, Kochsalalösung oder Luft geschehen, noch keineswegs erwiesen ist. Der einfache Lederhautstich ist das älteste operative Verfahren. Nach einer Mitteilung meines hochrerehrten Lehrers Hirschberg ${ }^{4}$ ), ist er zuerst ron J. Sichel

1) $0 \mathrm{hm}$, J., Über die Behandlung der Netzhautablösung dureh operative Entleerung der subretinalen Flüssigkeit und Einspritzung von Luft in den Glaskörper. v. Graefe's Arch. f. Ophth. Bd. LXXIX, 3. 1911.

2) Krusins, Zur Frage der Behandlung der Netzhantablösung durch Luftinjektion in den Glaskörper. v. Graefe's Arch. f. Ophth. Bd. LXXX, 2. 1912.

s) Rohmer, Effets des injections d'air stérilisé dans le vitré contre le décollement de la retine. Arch. d'opht. T. XXXII. p. 257-274.

4) Hirschberg, J., Zur operativen Behandlung der Netzhautablösung. Zentralbl. f. prakt. Augenheilk. 1891. S. 294 u. ebenderselbe, Behandlung der Kurzsichtigkeit. Urban u. Schwarzenberg, Berlin 1910. S. 54-58. 
im Jahre 1859 geübt und ron Alt, Wecker, Coccius, A. Graefe, Wolfe und Hirschberg selber empfohlen. Hirschberg nennt das Verfahren ungefährlich und vernünftig, sagt aber bei Gelegenheit der Mitteilung eines glïcklichen Falles „,allerdings ist Vollheilung sehr selten“1). Die unbefriedigenden Resultate erklären sich zum Teil meines Erachtens daraus, dass bei der einfachen Punktion mit dem Starmesser nur so viel der subretinalen Flüssigkeit ausfliesst, als unter dem Druck der gespannten Bulbushüllen steht. Ein um so grösserer Rest muss zurückbleiben, je ausgedehnter die Ablösung ist. Das von Hirschberg empfohlene Ansaugen durch zeltdachförmiges Abziehen der Bindehaut über der Punktionsstelle fördert die Entleerung wohl, macht sie aber doch nicht zu einer genügend ausgiebigen. Das Heraussaugen mit der Spritze oder auf die kïrzlich von Fuchs ${ }^{2}$ ) empfohlene Methode bewirkt eine zu plötzliche Entleerung, der schnell eine Wiederbildung der subretinalen Flüssigkeit folgen kann. Es fehit die Dauerwirkung, wie sie der Druckverband erzielt. Dieser plattet das punktierte Auge von vorn nach hinten ab; der Inhalt des Bulbus wird verkleinert, der Glaskörper drückt die Netzbaut unter Glätten der Falten gleichmässig und dauernd gegen die Sklera, und der Rest der subretinalen Flüssigkeit wird herausgepresst. So wenig verständlich die Wirkung des Druckrerbandes auf die Netzhautablösung bei intalkter Bulbuskapsel ist, so einleuchtend ist sein günstiger Einfluss, wenn das Auge punktiert, d. h. an der Stelle der Ablösung offen ist.

Die Punktion geschieht, während der Patient sitzt, mit einem breiten Starmesser an der Stelle der stärksten, natürlich gesenkten Ablösung durch meridionales Einstechen und Drehen des Messers um 90\%. Nach Abfliessen der subretinalen Flïssigkeit und Ansaugen durch zeltdachförmiges Abheben der Bindehaut iuber der Punktionsöffnung wird die Augenhöhle sorgsam mit Wattekugeln ausgefüllt, bis die Kuppe des Watteberges weit die Ebene des Orbitalrandes überragt, so dass der Orbitalrand das Auge nicht mehr vor Druck schützen kann. Eine Mullbinde presst dann mit jedem Bindenzuge den Bulbus fester in die Orbita, und eine Stärkebinde verhindert die Lockerung.

Dieser Druckverband wird meist gut vertragen. Öfters treten

x) Loc, cit.

2) Wien, ophth. Ges, 27. XI. 11. Klin. Monatsbl. f. Augenheilk. Bd, L, 1. S. 122. 1912 . 
nach einigen Stunden Schmerzen auf, die aber nur bei empfindlichen Patienten zum Verbandwechsel zwingen, und gewöhnlich bald wieder schwinden. Nach 24 Stunden wird der Verband ernenert. Die Hornhaut zeigt meist streifenförmige Trübungen, die die Ophthalmoskopie erschweren, aber keine Kontraindikation sind, den Druckrerband in derselben Weise wieder anzulegen. Am 3. Tag wird der Verband etwas weniger fest, am 4. Tag noch leichter, und am 5. Tag ein gewöhnlicher Verband angelegt, da die Skleralwunde nunmehr geschlossen ist, und am Verband sich keine ausgepresste subretinale Flïssigkeit, wie an den ersten Tagen mehr findet. Im Fall der Wiederanlegung der Netzhaut folgt dam noch eine Tiegekur von 3-1 Wochen obne weitere Massnahmen.

Vom Frühjahr 1908 bis zum März 1913 habe ich auf diese Weise 33 Fïlle schwerer Netzhautablösung behandelt. Es sind 22 Männer und 11 Franen im Alter von 16-73 Jahren. Bei 16 Patienten bestand excessire Myopie, bei 7 Myopie leichten oder mittleren Grades, and bei 10 Emmetropie. In 5 Fällen war ein Trauma vorangegangen, in 1 mit Perforation and Glaskörperverlust. 4 Fälle betrafen myopieoperierte Augen. In 3 Fällen bestand zur Zeit der Operation die Ablösung 4 Wochen, in 18 Fällen 2-3 Monate, in 6 Fällen ungefäln ${ }_{1 / 2} \mathrm{Jahr}$ und in 6 Fällen $1 \mathrm{Jahr}$ und länger.

Die Operation wurde in 19 Fällen nur 1 mal, in 11 Fällen 2 mal und in 3 Fällen 3 mal gemacht. Bei diesen 50 Punktionen war 22 mal bei der ersten Ophthalmoskopie nach der Operation vollständiges Anliegen der Netzhant festzustellen, 16 mal bestandeu noch Spuren von Ablösung, und $13 \mathrm{mal}$ fanden sich noch beträchtlichere Partien abgelöster Netzhaut; immer aber war die Ablösung erheblich kleiner geworden, inmer waren die bläulichen Buckel verschwunden. Da, wo die Ablösung nicht völlig beseitigt war, nahm sie meist in den folgenden Wochen wieder an Ausdehnung zu, um in mehreren Fällen allmählich den alten Zustand wieder zu erreichen. Bei der Mehrzahl dieser wurde der Eingriff ein oder mehrere Male zum Teil mit Friolg wiederholt. In den Fällen, in denen sich die Netzhaut völlig angelegt hatte, ist $12 \mathrm{mal}$ ein Rückfall erfolgt, und zwar 2 mal schon nach 8-14 Tagen, 2 mal nach 3-4 Wochen, 4 mal nach 6-8 Wochen, 3 mal nach 3-4 Monaten und 1 mal erst nach $\left.3^{1}\right|_{2}$ Jahren. In den übrigen 10 Fällen ist bisher Kein Rückfall beobachtet.

Damit der Leser sich ein eigenes Urteil ubber das Verfabren bilden kamn, halte ich es für angebracht, sämtliche Krankengeschichten, wenn 
auch in aller Kürze mit einigen epikritischen Bemerkungen anzuführen.

Fall 1. 24jähriger Arbeiter B. Im 10. Lebensjahr beiderseits Staroperation. Im Sommer 1907 Auftreten von Sehstörung. Dez. 1907 Aufnahme. Stat.: Aphakie $S$ R. $+9 D=\left.{ }^{5}\right|_{50}, \mathrm{~L} .+9 D=$ Handbewegungen. O. R. Alache, L buckelförmige Netzhautablösung der ganzen unteren Hälfte. 11. I. 08. Punktion mit Druckverband links. 1. IV. 08. R. Ablösung unverändert, L. flacher und Sehschärfe besser: R. $+9 D=55_{50}$, L. Finger in $1^{1 / 2} \mathrm{~m}$. Gesichtsfeld: R. oben innen bis $35^{\circ}$ beschränkt, L. konzentrisch beschränkt besonders oben und innen bis $20^{\circ}$. 1. IX. 08 . Wiederaufnahme wegen Verschlechterung der Sehkraft. $S$ R. und L. = Finger in $1 / 2 \mathrm{~m}$. 0 . beiderseits flache, aber fast totale Ablösung. Nach vergeblicher friedlicher Behandlung 18. IX. 08. Punktion mit Druckverband rechts. 20. IX. 08. Ablösung noch vorhanden. 16. XI. 08. $S$ R. $={ }^{5} / 60$, L. $=$ Finger in $2^{1 / 2} \mathrm{~m}$. Mit gebesserter Sehkraft entlassen. 21. XII. 09. $S \mathrm{R} .+12 D={ }^{5} /{ }_{55}, \mathrm{~L}$. $=$ Finger in $1 \mathrm{~m} .0$. R. hochgradige Ablösung, die nur die zentrale Gegend und den oberen äusseren Quadranten freilässt, L. Ablösung der unteren Hälfte.

Es handelt sich um einen Fall, der von vornherein prognostisch sehr ungünstig zu beurteilen war. Es ist möglich, dass die Katarakt schon Folge einer Glaskörpererkrankung war, es ist aber auch möglich, dass bei der Operation der angeborenen Kätarakt seinerzeit ein grösserer Glaskörperverlust geschehen ist. Die Ablösung des linken Auges bestand sicherlich schon Jahre lang, als das rechte Auge erkrankte und den geistig beschränkten Patienten schliesslich zwang, Aufnahme im Krankenhause zu suchen. Als das rechte Auge operiert wurde, war auch hier die Ablösung schon mindestens 5/4 Jahr alt. Die Operation wurde auf jedem Auge nur einmal gemacht, da man sich zu wenig von einer Wiederholung versprach. Funktionell war eine Besserung zu konstatieren, objektiv ist nichts erreicht.

Fall 2. Arbeiter W., 25 Jahre alt, Aufnahme 18. II. 1908 wegen Sehstörung auf dem linken Auge, die sich 14 Tage zuvor ohne bekannte Ursache eingestellt hat. $\mathrm{S}$ R. und L. $+0,5 D=5 / 5^{\circ}$ Gf. L. sektorförmiger Ausfall des oberen äusseren Drittels bis nahe $\left(8^{\circ}\right)$ zum Fixierpunkt. 0 . ausgedehnte Ablösung der unteren Hälfte bis an den Sehnerven mit tumorähnlicher Buckelbildung im unteren inneren Quadranten. Nach 10 wöchiger vergeblicher friedlicher Therapie mit Jodkalium, Schwitzen and subconjunctivalen Kochsalzeinspritzungen wird eine Punktion mit der Pravazspritze gemacht und ungefähr $1 / 5 \mathrm{ccm}$ der subretinalen Flüssigkeit entleert. 8 Tage später im wesentlichen dasselbe Bild wie vor der Aspiration. Deshalb am 18. V. 08. Punktion mit Druckverband. Danach Ablösung wesentlich verringert, aber noch nicht verschwunden. 22. VI. 08. Deshalb Wiederholung der Punktion mit Druckverband, die zur voliständigen Heilung führt. Anfangs sind noch Spuren flacher Ablösung nachweisbar, die aber im Laufe 
der nächsten Woehen ganz schwinden. 4 Monate nach der letzten Punktion stellte sich der Patient wieder vor mit vollkommen anliegender Netzhaut, die an Stelle đer früheren Ablösung nur scheckige Verfärbung zeigt, normaler Sehkraft und bis auf leichte Beschränkung aussen oben normalem Gesichtsfeld.

Wir haben hier einen Fall von Ablösung in einem emmetropischen Auge. Monatelange friedliche Behandlung nützte ebensowenig wie die einfache Punktion mit der Pravazspritze. Nach der ersten Punktion mit Druckverband Besserung, nach der zweiten Heilung der Ablösung, die nach 4 Monaten noch konstatiert werden konnte.

Fall 3. Frl. P., 23jährige Korrespondentin. Excessive Myopie. 1903 Myopieoperation links (Prof. Hirsehberg). Sehschärfe danach gut. 1905 Nachstardiscission. 4. III. 1908 Aufnahme auf der Augenabteilung des Virchow-Krankenhauses, da seit einigen Tagen hochgradige Sehstörung. des operierten Auges besteht. Stat. L. Spannung herabgesetzt. Pupille rund und frei. $S \mathbf{R}$. $-20 D=5 / 30$ fast, $\mathrm{L}$. $=$ Handbewegungen. Gf. nieht mehr aufzunehmen. 0 . ausgedehnte buekelförmige Ablösurug der oberen Hälfte bis zum Sehnerven reichend und den Sehnerven überhängend. Unter friedlicher Behandlung mit Bettruhe, Jod und subconjunetivalen Kochsalzeinspritzungen senkt sich die Ablösung, es werden Finger dieht vor dem Auge erkannt, und die Spannung wird gut. Am 14. IV. 08, 6 Wochen nach Eintreten der Ablösung, da auf friedlichem Wege keine weitere Besserung mehr zu erwarten ist, Punktion mit Druckrerband. Einige Tage nach der Operation Spannung etwas erhöht, so dass Eserin notwendig. Bei der ersten Ophthalmoskopie am 28. IV.08 nur noch Spuren von Ablösung sichtbar und $S=$ Finger in $21 / 2 \mathrm{~m} .4$. V. 08. $S=5 / 50$ and von Ablösung: nichts mehr zu sehen. 18. V. 08 als geheilt entlassen. $S+3 D$ eyl. ${ }_{45^{\circ}}{ }^{\circ}=\left.{ }^{5}\right|_{20}$. Gf. nur leicht konzentrisch beschränkt. Letzte Vorstellung am 12. III. 1912: $S$ R. $-22 D={ }^{5} /_{25}$. L. $={ }^{5} / 20-5 / 15,+5 D=$ Sn. 2 . O. Netzhaut liegt überall an. Also Heilung noch nach 4 Jahren zu konstatieren.

Dieser Fall stellt darum ein besonderes schönes Resultat dar, weil die Netzhautablösung nach Myopieoperation gewöhnlich für deletär gilt, ferner weil er nach 4 Jahren als ein Fall von Dauerheilung angesehen werden kanu. Wahrscheinlich bat die Drucksteigerung, die nach Schluss der Skleralpunktionswunde auftrat, zur Heilung mit beigetragen.

Fall 4. 44 jähriger Händler O. Myopia excessiva. Aufnahme 8. V. 08 wegen seit 2 Monaten bestehender hochgradiger Sehstörung links. Bisher mit strenger Bettruhe behandelt. Stat. $S \mathrm{R} .-12 D=5 / 10 . \mathrm{L} .=$ Finger in $8 / 4 \mathrm{~m}$. Gf. L. von oben beschränkt bis $20^{\circ}$.

O. L. reichliche Glaskörpertrübung, Angengrund dadureh stark verschleiert. Ablösung der ganzen unteren Netzhauthälfte bis an den Sehnerven in erhabenen bläulichen Falten. 
3 Wochen lang Behandlung mit Bettruhe, Schwitzen, Jod und Kochsalzeinspritzungen unter die Bindehaut ohne Erfolg. Dann 25. V. 08. I. Punktion mit Druckverband. 26. V. nur in der Peripherie noch eine Aach abgehobene Partie." 15. VI. 08. Ablösung wieder ausgedehnter. 22. VI. II. Punktion. 24. VI. Ablösung wieder erheblich verringert aber nieht fort. 7. VII. Ablösung wieder vergrössert. 8. VII. III. Punktion. 10. VII. Ablösung bis auf Spuren gesehwunden. 18. VII. Ganz fache Ablösung wieder da. 30. VII. 08. Ablösung ganz flach, aber doch wieder fast die ganze untere Hälfte des Augengrundes betreffend. Sehschärfe immerhin wesentlich gebessert $S \mathrm{R} .-12 \mathrm{D}=5 / 10^{\circ} \mathrm{L}=$ Finger in $4 \mathrm{~m} \mathrm{bzw.}=3{ }_{50}$. Gesichtsfeld nur leichte Beschränkung von oben bis $40^{\circ}$.

Ein Fall, in dem die Operation dreimal gemacht worden ist. Jedesmal schwand die Ablösung bis auf geringe Reste, um sich aber immer bald wieder auszudehnen. Nach der letzten Operation blieb sie nur flach, and das Endresultat war wesentliche Besserung der Sehschärfe und des Gesichtsfeldes. Ob diese von Dauer war, ist wegen baldiger Rückreise des Patienten in seine russische Heimat nicht zu sagen.

Fall 5. 19 jähriger Handelsmann L. Myopia magna. Vor $\left.3\right|_{4}$ Jahren Hufschlag gegen das linke Auge, seit 3 Wochen Sehstörung links. Aufnahme 9. V. 08. $S$ R. $-8 D=5 / 15$. L. $-8 D=$ Finger in $1 \mathrm{~m}$. Gf. L. Fehlen der oberen Hälfte bis $20^{\circ}$.

0. L. Ablösung der ganzen unteren Hälfte bis zum Sehnerven reichend. Therapie besteht zunächst in Gebrauch von Jod, subeonjunctivalen Kochsalzinjektionen und Schwitzen. Da keine Besserung, 25. V. Punktion mit Druckverband. 3. VI. 08 nur noch Spuren von Ablösung. 25. VI. 08 Ablösung wieder ausgedehnter. 29. VI. 08. Wiederholung der Operation. 30. VI. Ablösung kaum noch zu entdecken. 28. VII. 08 entlassen mit flacher Ablösung $S$ L. $-8 D=3 / 60$ Gf. normal.

Der Fall ist ähnlich wie der vorhergehende: die Amotio wurde verkleinert, die Funktion erheblich gebessert; aber die Wiederanlegung nicht erzielt.

Fall 6. Frau M. 42 Jahr. Privatpatientin. Myopia excessiva. IX. 08. Auftreten von Netzhautablösung rechts, oben innen beginnend in der Umgebung eines Risses, sehnell sich vergrössernd, bis sie im Dezember fast total ist trotz strenger Bettruhe usw. Finger werden in nächster Nähe vor dem Auge nur mit Mühe erkannt. 3. I. 09. I. Punktion mit Druckverband 5. I. Ablösung wesentlich flacher, aber keineswegs verschwunden. 24. III. 09. Ablösung wieder total. 5. IV. 09. II. Punktion mit Druckverband. 8. IV. 09. Ablösang fort. Netzhaut liegt überall an. 21. IV. 09. Netzhaut bleibt anliegend $S \mathrm{R}-18 \mathrm{D}=\left.5\right|_{25}$ Gf. normal. 26. VII. 09 . als geheilt entlassen. 7. IX. 09. Patientin ist gegen ärztlichen Rat ins Seebad gereist. Gestern wieder plötzliches Auftreten eines Schattens vor dem rechten Auge. Beginnende Amotie ist nachweisbar oben inrien genau an der alten Stelle wie im IX. 08. 10. IX. 09 sebnelle Zunahme der Amotio 
29. IX.09. Ablösung wieder fast total. 25. X. 09. Erst jetzt entschliesst sich die Patientin zu einer nenenWiederholung der Operation. 27. X.09. Ablösung vollständig verschwanden. 13. XI. 09. Ablösung bleibt fort $s-19 D={ }^{55}$ Gf. normal 20. XIT. 09 neuer Rïckfall wieder oben innen. 30. XII. 09. Ablosung macht rapide Fortschritte. 20. II. 10. Amotio fere totalis. $S \mathrm{R} .=$ Finger in $3 / 4 \mathrm{~m}$. Patientin entschliesst sich nicht zu neuem Eingriff. 18. IX. 12. R. Strabismus divergens, Cataracta complicata S R.Handbewegung. L. $-18 D=5 / 15$.

Wenn auch in diesem Fall das Endresultat ein schlechtes ist, so ist er doch wie kaum ein anderer geeignet, die güntige Wirkung der Behandlungsmethode zu beweisen. Zweimal gelang es damit, eine seit Monaten bestehende total abgelöste Netzhaut sofort zur volligen Wiederanlegung zu bringen und dem erblindeten Auge die frühere Sehkraft wiederzugeben. Das erste Mal blieb das Auge 4 Monate, das zweite nur 2 Monate lang geheilt. Das Resultat der Operation war in dem absolut hoffuungslos scheinenden Falle beide Male ein so unerwartetes und iberraschend günstiges, dass es vor allem dieser Fall war, der mich veranlasst hat, diese Kombination von Punktion und Druckverband regelmässig bei Amotio zu versuchen.

Fall 7. Beamter R. 49 Jahre, Myopia excessiva. Aufuahme 4. VI. 08, wegen Verschleierung vor dem bessern rechten Auge seit 4 Wochen. Stat. Tension L. herabgesetzt. $S \mathrm{R}-14 D=5 / 30^{\circ}$ L. $-10 D=5 / 80^{\circ}$ Gf. L. Fehlen der oberen Hälfte bis $20^{\circ}$. 0 . R. Cataracta cortic. post. Glaskörpertribungen, periphere chorio-ret. Veränderungen. L. Glaskörpertrübungen. Ausgedehnte wenn auch flache Ablösung der ganzen unteren Hälfte. - Unter Bettruhe, Jod, subeonj. Na Cl-Einspritzungen Versehlechterung 28. VI. 08. $S={ }^{5} / 60.29$. VI. Panktion mit Druckverband. 14. VII. Wesentliche Besserung, $\mathrm{Nur}$ in der unteren nasalen Hälfte noch flache Amotio. $S \mathrm{~L}$. $9 D=5 / 20$. Gf. annähernd normal. 1. VIIL. 08 nur in der Peripherie noch ganz leichte Fältelung der Netzhant, entlassen. 19. VI. 09. Allmählich hat sich die Sehschärfe links wieder verschlechtert. Heute Tension stark herabgesetzt. Grosse Blutung in die V.K. \& L. = Mandbewegung.

Ein von vornherein ungünstig zu beurteilender Fall, da die hintere Corticalkatarakt, die Glaskörpertruibungen und die peripheren chorioiditischen Veränderungen im rechten Auge auch links schwere ureitische Vorgänge vermuten liessen. Die Operation schaffte wesentliche Besserung. Dex spätere Ausgang in Erblindung aber unter intraocularen Blutungen und Weichheit des Augapfels machte die ursprüngliche Vermutung wahrscheinlich.

Fall 8. 40jähriger Maurer P. Vor 14 Jahren Lues. Früher beiderseits gut gesehen, nicht kurzsichtig. Vor 4 Jahren Erkrankung des reehten, vor 3 Jahren auch des linken Auges an Netzhautablösung. Vor 2 Jahren 
anderswo mit $H_{g}$-Kur in klinischer Behandlung. Danach Sehschärfe gebessert. Seit 1/ Jahr aber Sehschärfe wieder erheblich versehlechtert. Aufnahme 17. VI. 08. Stat.: Strabismus diverg. $S \mathrm{R} .=$ Finger in $3 \mathrm{~m} . \mathrm{L}$. $=$ Finger in $2 \mathrm{~m}$. Gf. R. Fehlen der oberen Halfte bis nahe zum Fixierpunkt und Einschränkung von unten bis $30^{\circ}$. L konzentr. Beschränkung besonders von oben und unten bis $25^{\circ}$. O. R. Cataracta incip., reichliche Glaskörpertrübung. Ganze untere Hälfte der Netzhaut abgelöst bis an den Sehnerven in bläulichen gardinenartigen stark prominenten Buckeln, in der Peripherie der oberen Hälfte flache Ablösung; da wo die Netzhaut noch, bzw. wieder anliegt, zahlreiche Streifen und Pigmentiernngen. $4 \mathrm{~Pa}$ oberhalb des Sehnerven auf der Netzhant ein bläuliches Exsudat mit Resten von Blut. Emmetropie. L. ist wegen vorgesehrittener Katarakt kein Bild vom Augengrunde zu bekommen. Zunächst 4 Wochen lang Schmierkur, Bettruhe und subconj. Kochsalzeinspritzungen. Dabei weitere Verschlechterung. $S R$. $=$ Finger in 1 m. 17. VII. 08. Punktion mit Druckverband R. 25. VII. 08. Die bläulichen Falten sind geschwunden. 28. VII. Die Netzhaut liegt an. Sehschärfe besser. 7. VIII. Ablösung kehrt wieder. 14. VIII. $s \mathrm{R}=$ Finger in $4 \mathrm{~m}$. Ablösung wieder stärker. 21. VIII. Ablösung fast wieder so ausgedehnt wie vor der Operation. $H_{g}$-Kur. 3. IX. 08. Wiederholung der Operation wieder nur mit vorübergehendem Erfolg. Patient wird am 5. XII. 08 ungeheilt entlassen.

Ein ganz veralteter, verzweifelter Fall doppelseitiger Ablösung in emmetropischen Augen, bei dem wahrscheinlich Lues eine ursächlich Rolle spielte. Nach der ersten Operation gelang es, vorübergeheud die Netzhaut zur Wiederanlegung zu bringen. Das Endresultat war schlecht.

Fall 9. 24jähriges Dienstmädchen K. Myopia magna, Seit einigen Tagen hochgradige Sehstörung links. Aufnahme 22. IV. 08. Stat.: Rechts Spannung herabgesetzt. $S$ R. $=$ Handbeweg. L. $-12 D=5$. Gf. Fehlen der nasalen Hälfte bis über den Fixierpunkt. O. L. erhabene Ablösung der ganzen äusseren Hälfte der Netzhaut bis nahe an den Sehnerven. Ther: zunächst Jodkalium, Schwitzen, subconj. Kochsalzeinspritzungen. Nach teilweiser Senkung der Ablösung am 18. V. 08. Panktion mit Druckverband: 19. V. 08. Fon Ablösung nichts mehr zu sehen. 25. V. 08. Netzhaut liegt überall an. Sehschärfe gut. 27. V. Patientin empfindet seit heute nasal wieder einen Schatten, flache Ablösung unten aussen wieder da. 9. VI. Ablösung wieder ausgedehnter, wenu auch flach, $S$ R. $-9 D=5 / 50$ Gf. nasale Beschränkung bis $35^{\circ}$ 13. VI. 08 aus äusseren Gründen entlassen. 22. VI. 08. Wiederanfnahme $S$ R. $-10 D=5 / 50$. Gf. nasale Beschränkung bis $45^{\circ}$. O. noch flache Ablösung der oberen Hälfte. 20. VII. 08. Punktion mit Druckverband. 5. VIII. 08. Ablösung erheblich zurückgebracht. 15. VIII. $S$ R. $-9 D={ }^{5} / 50$ Gf. nasale Beschränkung bis $40^{\circ}$. O. Netzhaut im wesentlichen anliegend.

Das Resultat-ist in diesem Fall befriedigend zu nennen; eine hochgradige Ablösung, die Erblindung des Auges bewirkt hatte, 
ist durch zwei Operationen bis auf geringe Reste beseitigt, und ein brauchbares Sehrermögen ist wiedergewonnen. Leider ist die Patientin aus dem Auge verloren, und über ihr weiteres Schicksal nichts bekannt.

Fall 10. 25jähriger Techniker K. Myopie levis. Anfnahme am 1. VII. 08 wegen eines dichten Schleiers, der seit zwei Tagen vor dem rechten Auge besteht. Stat.: $S$ R. Finger in $1 / 12 \mathrm{~m}$. L. $-0,5 D=5 / 5$. Gf. Fehlen des oberen inneren Drittels fast bis zum Fixierpunkt. O. R. Ansgedehnte buckelförmige $A$ blösung der ganzen Schläfenhälfte. Vier Wochen lang vergebliche Behandlung mit Sehwitzen, Jod, Kochsalzeinspritzung und Bettruhe. 27. VII. Punktion mit Druckverband 5. VIII. Keine Amotio melr nachweisbar. 25. VIII. $S$ R. $=\left.{ }^{5}\right|_{20}$. Gesichtsfeld normal. 0 . Netzhaut liegt vollkommen an. Entlassen. 8. IX. 08. Wiederaufnahme wegen Rï ckfalls der Ablösung, $s R_{.}={ }^{5}{ }_{20}$. Gf. normal. 0 . unten aussen umschriebene Aache Ablösung. Nach 14 tägiger sogenannter conservativer Behandlung Ablösung wesentlich fortgeschritten $S 5 / 50$ Gf. Beschränkung oben inmen bis $20^{\circ}$, deshalb am 24. IX. 08. II. Punktion mit Druekverband. 26. IX. 08. Ablösung fort. 2. XI. Keine Spur von Ablösung mehr sichtbar $S$ R. $1,5 D$ cyl. $=5 / 15$ Gf. normal. Der Patient wird in den folgenden Jahren regelmässig gesehen, die Ablösung bleibt fort. Am 7. VI. $13 \mathrm{~S}$ R. $0,75 \mathrm{D}$ eyl. $={ }^{5} / 7$ Gf. normal. Die Netzhaut liegt überall an,

Das Resultat ist in diesem Fall besonders günstig: die erste Operation brachte vorübergehende, die Wiederholung dauernde Heilung einer fast deletär scheinenden Netzhautablösung mit Wiedergewinnen der vollen Funktion. Nach fast 5 Jahren hat man das Recht, von Dauerheilung zu sprechen.

Fall 11. 20jähriger Handlungsgehilfe S. Im achten Jahr fast gänzliche Erblindung des rechten Auges. Anderswo mehrfach vergeblich behandelt. Aufnahme am 5. I. 08 wegen Sorge um das linke Auge, das immer gut gesehen hat. $S \mathrm{R}=$ Finger in $75 \mathrm{~cm}, \mathrm{~L} .+2 D={ }_{5}{ }_{5}$. Gf. Fehlen der oberen Hälfte bis weit über den Fixierpunkt. O. R. Ablösung der ganzen unteren Hälfte bis an den Sehnerven und über die Mitte reichend, in der oberen Hälfte alte Pigmentherde und Streifen als Zeichen, dass auch hier Ablösung bestanden hat. L. alles normal. Konservative Behandlung erfolglos. 25. I. 08 einfache Punktion. 9. II. 08. Ablösung etwas flacher, sonst unverändert. $s R$. = Finger in $1 \mathrm{~m}$, entlassen. 3. VII. 08. Wiederaufnahme wegen Schmerzen im rechten Auge. Spannung herabgesetzt. Bild der Ablösung wie im Jan. 08. ४. VII. Punktion mit Druekverband: 17. VII. Keine Ablösung melr zll sehen. 1. VIII. 08. $S$ R. Finger in $50 \mathrm{~cm}$. Von Ablösung kaum noch etwas nachzuweisen. Am 11. I. 10 stellt Patient sich wieder vor. Keine Spur vou Ablösung sichtbar. Netzhaut aber atrophiseh, und $s$ schlecht $=$ Finger in $1 / 2 \mathrm{~m}$.

In diesem Fall ist eine seit 12 Jahren fast total abgelöste Netzhaut durch die Operation zur Wiederanlegung gebracht. Thre Ent- 
Die Kombination von Punktion und Druckverband zur Behandlung usw. 347

artung war, wie zu erwarten, so weit vorgeschritten, dass eine funktionelle Besserung nicht mehr erzielt werden konnte.

Fall 12. 24jähriger Arbeiter W. Hochgradig kurzsichtig seit Kindheit. 1899 Myopioperation links anderswo, 1902 Netzhautablösung, die später zu Glaukom führte und schliesslich die Enucleation nötig machte. 23. IX. 08. Aufnahme wegen Sehstörung rechts, die vor einigen Tagen nach Heben schwerer Lasten aufgetreten ist. $S \mathrm{R} .-10 \mathrm{D}=$ Finger in $1 \mathrm{~m}$. Gf. stark konzentrisch beschränkt. 0 . Glaskörpertrübung. Ablösung der ganzen unteren inneren Häfte. 9. X. Nach 15 tägiger erfolgloser Bettruhe, Punktion mit Druekverband. 19. X. Zentrum frei von Ablösung, Sehschärfe besser, unten aussen noch beträchtliche Ablösung, 21. X. Auftreten von blasiger Ablösung in der oberen Hälfte. 23. X. fast totale Ablösung, IT. Punktion mit Druckverband. 24. X. nur geringe Besserung. 13. XI. III. Operation. 23. XI. Keine Besserung. 18. I. 09 mit fast totaler Ablösung und Sehschärfe - Handbewegungen entlassen.

Im vorliegenden Fall haben drei Operationen gänzlich versagt, und der Patient ist erblindet.

Fall 13. Fränlein B. 19 Jahre. Tochter eines Arztes. Myopia excessiva. Erste Vorstellung am 10. XI. 08, da seit 10 Tagen hochgradige Sehstörung rechts besteht. $S \mathrm{R} .-16 \mathrm{D}=$ Finger in $3 \mathrm{~m}, \mathrm{~L} .-14 D=5 / \mathrm{r}$. Gf. rechts nur mässig beschränkt. $O$. R. flache, aber fast die ganze obere Hälfte betreffende Ablösung. 8 Wochen lang strenge Bettruhe, Schwitzkur und subeonj. Kochsalzeinspritzungen, ganz ohne Erfolg. Die Ablösung hat sieh gesenkt, sie betrifft die ganze untere und einen Teil der oberen Hälfte. 7. I. 09. Punktion mit Druckverband. 12. I. 09. Keine Spar von Ablösung mehr. 30. III. 09. Ablösung bleibt fort. 8 R. $-14 D=5 / 25$ Gf. normal. 10. IV. 09 geheilt in die Heimat entlassen. 18. IV. 09. Plötzliches Wiederauftreten von Sehstörung. Rückfall der Ablösung unten aussen an umschriebener Stelle. 12. V. 09. II. Operation. 15. V. Ablösung flacher aber nicht fort. 25. VI. 09. III. Operation. 1. VII. flache Ablösung besteht noch in der Peripherie. 24. VIII. 09 mit gebesserter $S-10 D=4 / 35$ und subjektiven Woblbefinden entlassen. Anfänglich bielt sich die Sehschärfe auf dieser Höhe, in letzter Zeit ist sie wieder schlechter geworden bei Ausdehnung der wenn auch flachen Amotio bis zum Sehnerven. 3. VI. 13. $S$ R. $-6 D=1 / 25$. Gf. Beschränkung von oben innen bis $20^{\circ}$.

Das Resultat ist nicht so unginstig. Die erste Operation führte für $3^{1 /}$ Monate zur Wiederanlegung der fast total abgelösten Netzhaut, deren Behandlung zurzeit von einem unserer ersten Klimiker für aussichtslos hingestellt.wurde. Zwei Wiederholungen der Operation brachten wohl Besserung, aber keine Heilung. Wenn auch in letzter Zeit eine allmähliche Verschlechterung eingetreten ist, so ist doch 41/2 Jahre nach Beginn der Erkrankung das Auge noch brauchbar, und die Patientin, die inzwischen geheiratet hat, fublt sich durch ihr Leiden nur wenig gestört. 
Fall 14. Frau Rittergutsbesitzer Sch. 22 Jahre. XI. 07. Sturz von der Treppe, bald danach Auftreten eines Schattens vor dem rechten Auge. Erste Konsultation 30. XII. 07. S R. $-11 \mathrm{D}={ }^{5} / 20$, L. $-11 D=5 / 6^{\circ}$. Gesichtsfeld rechts zentrales Skotom. 0 . rechts fast mikroskopische zentrale Ablebung der Netzhantmitte. Nach strenger Bettruhe usw. Wiederanlegung und Besserung der Funktion. 25. IX. 08 kehrt sie wieder mit facher, aber ausgedehnter faltiger Ablösung der ganzen unteren Hälfte bis an den Sehnerven heranreichend $S R .-9 D=\left.{ }^{5}\right|_{25}$. Gf. Beschränkung von oben bis $10^{\circ}$. Sofort Aufnahme. Nach vierwöehiger vergeblicher konservativer Behandlung: 26. X. 08. Punktion mit Druckverband. 1. XI. 08. Ablösung beseitigt. Acht Wochen später mit vollkommen anliegender Netzhaut, $S=5 / 15$ und normalem Gesichtsfeld nach Hause entlassen. Ein Jahr später 30. XI. 09 $S \mathrm{R}-11 \mathrm{D}=-2 \mathrm{D} \mathrm{cyl} \rightarrow=\left.5\right|_{15}$. Gesichtsfeld normal. 0 . keine Spur von Ablösung. Dieser erfrealiche Befund konnte bei den regelmässigen Untersuchungen in den folgenden Jahren immer wieder festgestellt werden, bis die Patientin im April 1912 wiederkelirte mit der Angabe, seit kurzem eine Sehstörung auf dem rechten Auge wieder zu bemerken. $S R=5 / 95^{\circ} 0$. unten innen flache Ablösung nachweisbar bei noch annähernd normalem Gesichtsfelde. Also Rückfall nach $31 / 2$ Jahren. Die Patientin konnte sich bisher zu einer Wiederholung der Operation nicht entschliessen, zumal weitere Verschlechterung bis heute nicht eingetreten ist.

Einmalige Punktion mit folgendem Druckverband hat hier zur völligen Wiederanlegung der zur Hälfte abgelösten Netzhaut geführt. $3^{1 / 2}$ Jahr lang blieb das Auge gesund und leistungsfähig, und der Fall wurde als Fall von Dauerheilung gefuihrt. Wenn auch jetzt ein Rückfall eingetreten ist, so muss der Fall doch zugunsten der angewandten Methode sprechen, durch deren Wiederholung eine neue Wiederanlegung der einen Quadranten betreffenden Netzhautablösung zu erhoffen ist.

Fall 15. Frau J. 53 Jahre. Kurzsichtig seit Kindheit. Vor 3 Jahren Erblindung rechts durch Netzhautablösung. Aufnahme 29. XII. 08 wegen Sehstörung links seit 8 Tagen. Stat. R. Cataracta compl. $S \mathrm{R} .=$ Handbewegung. L. = Finger in $1 \mathrm{~m}$. Gesichtsfeld starke konzentrische Beschränkung. 0 . links buckelförmige Ablösung der ganzen oberen äusseren Hälfte den Sehnerven überhängend. - In den folgenden Wochen Senkung der Ablösung ohne Wiederanlegung oben, so dass sie bald fast total ist. 2. II. 09. Operation. Besserung nur beschränkt, 1. III, 09 wieder fast totale Ablösung. 2. III. 03. II. Punktion. 4. III. 09. Auffallende Besserung, nur unten aussen noch flache Ablösung. 24. IIr. 09. Ablösung wieder im Wachsen begriffen, deshalb III. Punktion. Auch diese bringt nur partielle Besserung. 24. IV. 09. entlassen mit $S L_{\text {. }}=1 / 15$ und annähernd normalem Gesichtsfeld, dabei besteht noch ziemlich ausgedehnte Abiösung unten und oben aussen.

Fall 16. Arbeiter K. 50 Jahre. Rechts Erblindung im zelinten Jahre. Links immer kurz- und schwachsichtig. Seit Anfang Januar 09 starke Versehleierung ror dem linken Auge. Aufnahme 26. I. 09. Stat. Rechts 
Staphyloma corneae, queres Kalkband $S=0$. Links degenerative Verdünnung und Retraktion der Iris. $S=$ Finger in $1 / 2 \mathrm{~m}$. 0 . links fast totalo Netzhautablösung. 11. II. 09. Punktion mit Druckverband. 13. II. 09. Patient gibt beim Verbandwechsel an, dass die Sehschärfe besser geworden ist. Tatsåchlich sind die gardinenartigen Buckel verschwunden und nur noch flache Ablösung ist vorhanden. 27. II. 09. das Bild ist im wesentlichen wieder dasselbe wie vor der Operation. Sehschärfe $=$ Finger in $1 \mathrm{~m}$ bei mässig konzentrisch beschränktem Gesichtsfelde. Patient wird entlassen.

Diese beiden Fälle betreffen Patienten mit fast totaler Ablösung, des einzigen Auges. Die an und für sich schlechte Prognose verschlechterte sich noch in dem ersten Fall mit Rücksicht auf den malignen Verlauf der Ablösung des ersterkrankten Auges und im zweiten wegen Degenerationserscheinungen an der Iris des zu behandelnden Auges. Im ersten Fall hat eine dreimalige Operation nur wenig, im zweiten eine einmalige garnichts genützt.

Fall 17. Frau B. 50 Jahre. Aufnahme 14. VII. 09. Immer kurzsichtig gewesen, seit 14 Tagen Sehstörung rechts, $S \mathrm{R}=55_{50}$ L. $-5 \mathrm{D}$ $=5 / 10$. Gesichtsfeldbeschränkung von oben bis $10^{\circ}$ und unten aussen bis $60^{\circ}$. O. rechts fast totale Ablösung. Links normal. 22. VII. 09. Punktion mit Druckverband. 25. VII. 09. Die Netzhantbuckel sind verschwunden, nur flache Ablösung besteht noch. 17. VIII. 09 gebessert mit Sehschärfe = $\left.1\right|_{10}$ entlassen. 24. VIII. 09. Die Ablösung hat bereits wieder an Ausdehnung gewonnen.

Fall 18. Maler A. 38 Jahre. Früher beiderseits gut gesehen. Vor drei Wochen Faustschlag gegen das linke Auge; seit fünf Tagen ziemlich plötzlich aufgetretene völlige Erblindung dieses Auges. Stat. $S$ R. $=5 / 6$. L. $=$ Lichtsehein. O. rechts normal. Links fast totale Netzhautablösung. 29. I. 10. Punktion mit Druekverband. 3. II. 10. Ablösung bleibt bestehen, wenn auch in flacherer Ausdehnung. 15. II. 10 flache, aber allseitige Ablösung. $S=$ Finger in nächster Nähe. Wegen Delirium auf die Stat. der Unuruhigen verlegt.

Fall 19. Aufwärterin N., 46 Jahre. Myopia excessiva. Anfang April 1910 Sturz von der Treppe, danach Sehstörung durch frische Netzhautblutungen $S$ R. $-20 D=\left.5\right|_{50 .}$ L. $-20 D=\left.1\right|_{50}$. 25. IV. 10. Auftreten von Netzhautablösung oben aussen, die schnelle Fortschritte macht und Mitte Juni fast total ist. $S=$ Finger in $20 \mathrm{~cm}$. 20. VI, 10. Punktion mit Druckverband. 27. VI. 10 keine wesentliche Besserung. 2. VIII. 10. Patient wird ungeheilt mit etwas gebesserter $S=$ Finger in $1^{1 / 2} \mathrm{~m}$ entlassen.

Auch in diesen drei Fällen, von denen zwei traumatischen Ursprungs sind, bestand zur Zeit der Operation allseitige Ablösung und wenig Aussicht auf einen therapeutischen Erfolg. Die Behandlung war dementsprechend so gut wie erfolglos.

Fall 20. Schneider P. 48 Jahre. Myopia levis. Im VI. 09. Stoss mit Schirmspitze gegen das linke Auge, bald danach Abnahme der Sehschärfe 
und Funkensehen. Seit Anfang 1. 10 erhebliche Versehlechterung der Sehschärfe. Aufnahme 12. II. 10. $S$ R. $-1,5 D=5 / 5^{\circ}$ L. $=$ Finger in $30 \mathrm{~cm}$. Gesichtsfeld: Fehlen der unteren Hälfte. 0 . buckelförmige Ablösung der oberen Hälfte den Sehnerven überhängend. 15. III. 10. Nach vergeblicher friedlicher Behandlung und Senkung der Ablösung Punktion mit Druckverband. 10. III. 10. Ablösung völlig gesehwunden. 8. IV. 10. Netzhaut liegt äberall an $S=5 / 5$. Patient muss auf eigenen Wunsch gegen ärztlichen Rat entlassen werden.

Fall 21. Mechaniker F, 33 Jahre. Myopia media. Seit einem Jahr Sehstörnng rechts. Aufuahme 8. IV. 10. $S \mathrm{R}$. $-2,5 D={ }^{5} / 50$. L. $-5 D$ $=\left.{ }^{5}\right|_{6^{*}}$ Gesichtsfeldbeschränkung von oben aussen bis $20^{\circ}$, unten aussen bis $40^{\circ}$. 0 . rechts ansgedehnte Ablösung aussen unten. Glaskörpertribungen. 20. IV. 10. Punktion mit Druckverband. 22. IV. 10 nur in der äussersten unteren Peripherie ist noch etwas Ablösung nachweisbar. 26. IV. 10. Keine Ablösung mehr zu entdecken. 30.IV. 10. Netzhaut liegt überall an. 19. V.10 geheilt entlassen mit $S-2,5 D=\left.{ }^{5}\right|_{30}$ und annähernd normalem Gesichtsfeld.

In diesen beiden Fällen gelang es, durch eine Operation eine monatelang bestehende schwere Netzhautablösung zu beseitigen. Leider ist in beiden die Beobachtungszeit wach dex Operation nur kurz, im ersten nur drei, im zweiten vier Wochen, so dass sie nicht als Fälle von Heilungen längerer Dauer angeführt werden können.

Fall 22. Versichernngsbeamter R. 27 Jahre. Myopia magna. Aufnahme 21. VI. 10. Vor 14 Tagen Auftreten eines Schattens vor dem linken besseren Auge, seit vier Monaten schon Flockensehen. Stat. $S$ R. $-5 D=\left.{ }^{5}\right|_{30}$ fast, L. $-8 D=5{ }^{5}{ }_{30}$ fast. Gesichtsfeld links ron oben beschränkt bis 120 . 0 . links flache aber auggedehnte Netzhautablösung, die ganze untere Hälfte bis an den Sehnerven betrefiend; nasal erstreckt sie sich auch auf die obere Hälfte. Mehrere Netzhautrisse. Drei Wochen lange Behandlung mit strenger Bettruhe, Schwitzen, Jodkalium und subconjunctivalen Kochsalzeinspritzungen, dabei Fortschreiten der Ablösung deshalb 18. VII. 10. Punktion mit Druekverband. 22. VII. 10. Keine Spur von Ablösung mehr vorhanden. 10. VIII. 10. Netzhaut liegt überall an. 20. VIII. 10. Patient klagt wieder über das Vorhandensein eines Schattens. Leichte Ablosung wieder da. 29. VIII. 10. Die Ablösung hat wieder schnelle Fortschritte gemacht, deshalb Wiederholung der Operation. 2. IX. 10. Keine Spur von Ablösung mehr. Sehschärfe gut. Schleier fort. 19. XI. 10. Die Ablösung ist nicht wiedergekehrt. $S$ L. $-8 D=5 / 20$ Gesichtsfeld normal. 19. XII. 11. Letzte Vorstellung. $S L_{0}-8 D=5 / 20$ Gesichtsfeld normal. 0 . Netzhaut überall anliegend. Also geheilt nach 16 Monatea.

Im vorliegenden Falle ist die günstige Wirkung der Operation sehr augenscheinlich; unter strenger Bettruhe usw. lram es zum Fortschreiten der schon über die Hälfte abgeiösten Netzhaut, nach der ersten Operation zur Wiederanlegung aber nur fir 5 Wochen. Die zweite Operation erst schaffte dauernde Heilung; wenigstens konnte nach 16 Monaten noch die Heilung festgestellt werden. 
Fall 23. Kammaeher Sch. 32 Jahre. Immer gut gesehen, nicht kurzsichtig. Seit 7 Wochen Flimmern vor dem linken Auge, seit 4 Wochen Abnahme der Sehschärfe. Kein Trauma vorausgegangen. Aufnahme 30. VI. 10. $S \mathrm{R} .={ }^{5} /{ }_{6} . \mathrm{L} .={ }^{5} / 60$ Gesichtsfeld Beschränkung von innen bis $25^{\circ} .0$. Flache Netzhautablösung des unteren änsseren Quadranten. 27. VIII. 10. nach achtwöchiger friedlicher Behandlung Ablösung ausgedehnter bis an den Sehnerven heranreichend. Deshalb heute Punktion mit Druckverband. 10. IX. 10. Nur ganz flache Ablösung im äusseren unteren Quadranten besteht noch. 27. IX. 10. Ganz flache Ablösung unten aussen noch vorhanden. $S=55_{50}-\left.5\right|_{30}$ Gesichtsfeld relative Einschränkung bei herabgesetzter Beleuchtung bis an den Fixierpunkt, bei heller Belenchtung normal.

Die Prognose war hier relativ günstig zu stellen; es bestand keine Myopie, und die Ablösung war nur flach und partiell. Die konservative Behandlung wurde deshalb länger gewöhnlich als fortgeführt und erst nach acht Wochen die Operation rorgenommen. Dieselbe führte wohl zur Besserung aber nicht zur Wiederanlegung. Leider musste aus äusseren Gründen eine Wiederholung der Operation unterbleiben, die meines Erachtens aussichtsreich war. Über das spätere Schicksal des Patienten ist nichts bekannt.

Fall 24. Heizer D. 35 Jahre. 7. I. 1911. Sehwere perforierende Augenverletzung durch Glassplitter. Klaffende Skleralwunde mit Glaskörperverlust und Hämophthalmus. Nach Naht reizlose Heilung. 28. I. 11 entlassen mit $S \mathrm{~L}=5 / 20$. Gesichtsfeld normal. 0 . Glaskörperflocken, aber normaler Augengrund. 12. IV. 11. Wiederaufnahme wegen plötzlich aufgetretener Sehstörung. $S \mathrm{~L}$. $=$ Finger in 1/2 m. O. buckelförmige Netzhautablösung oben aussen. Diese macht rapide Fortschritte und ist bald total $S=\frac{1}{\infty}$ 1. V. 11. Punktion mit Druckverband. 2. V. 11. Buckel fort, nur noch flache Ablösung, Sehnerv siehtbar, Sehschärfe besser. 5. V. 11. Ein Buckel ist wieder entstanden. 10. V. 11. Ablösung wieder so ausgedehnt wie vor der Operation. 12. V. 11. II. Punktion. 22. V. 11 ungeheilt entlassen.

Es handelt sich hier um einen Fall ron Netzhautablösung nach Glaskörperverlust, die erfahrungsgemäss gewöhnlich zu schnellem und unabwendbarem Untergang der Sehkraft fuhrt. Dieser Fall steht daher etwas ausser der Reihe, und das ungünstige Resultat der Behandlung muss dementsprechend gewertet werden.

Fall 25. Lagerist J, 28 Jahre. Wegen Schwachsichtigkeit vom Militärdienst befreit. XII. 1910. Erkrankung an Netzhautablösung rechts. In verschiedenen Kliniken ohne Erfolg konservativ behandelt. Aufnahme 3. III. 11. R. Spuren sehr reichlicher subconjunctivaler Injektionen. $S \mathrm{R}$. $=$ Finger in $4 \mathrm{~m} . \mathrm{L} .={ }^{5} /{ }_{12}-2 D$ cyl. $\rightarrow={ }^{5} /{ }_{10}$. Gf. eingeschränkt bis auf ein zentrales Feld von $10^{\circ}$ and den unteren äusseren Quadranten. 0 . fast totale $A b$ lösung. 6. III. 11. Punktion mit Druckverband. 8. III. 11. Keine 
Spur von Ablösung mehr sichtbar. 14. III. 11. Hente in der Peripherie wieder beginnende Ablösung vorhanden. 20. III. 11. Ablösung wieder ausgedehnt. 24. III. 11. S R. $={ }_{3}^{2 /}$ entlassen.

Die Ablösung war alt und fast total. 8 Tage lang war nach der Operation keine Spur von Ablösung mehr nachweisbar, dann trat sie in der äussersten Peripherie wieder auf, um sich allmählich wieder, wenn auch längst nicht bis zu dem früheren Grade auszudehnen.

Fall 26. Schneider W, 55 Jahre, Myopia magna, Aufnahme 15. V. 11. Seit 5 Jahren schon Verseblechterung der Sehschärfe rechts und Fimmern, seit einigen Monaten weitere Abnahme der Sehschärfe. Spannung rechts herabgesetzt. $S \mathrm{R} .=$ Handbewegungen. L. $-9 D=5 / \%$. O. erhabene, fast totale Netzhautablösung. 29. V. 11. Punktion mit Druckverband. 4. VI. 11. Sehschärfe wesentlich gebessert. Keine Ablösung sichtbar. 10. VI. 11. Bläuliche Buckel bilden sich wieder. 22. VI, 11. Ablösung wieder hochgradig. $S=$ Finger dicht vor dem Auge. II. Pnnktion. 28. VI. 11 . Ablösung besteht noch. 8. VII. 11 ungeheilt entlassen.

Auch hier war die Ablösung alt und allseitig, auch hier gelang es nur für kurze Zeit, die Netzhaut zur Wiederanlegung zu bringen; bald trat ein Rückfall auf, der schnell Fortschritte machte. Der zweite operative Versuch war ganz erfolglos.

Fall 27. Kassenhote H., 47 Jahre alt. Myopia media. Aufnahme 15. VII. 11. Seit 3 Tagen Sehstörung rechts, zuerst aufgetreten nach anstrengendem Laufen und Aufspringen auf die elektrische Bahn; täglich Verdichtung des Schleiers. Seit $1 / 4 \mathrm{Jahr}$ schon Flimmern und Flockensehen. Stat: $S$ R. $=$ Finger in nächster Nähe. L. $-4 D=5 / 15$. Gf. Beschränkung von unten bis $10^{\circ}$ über Fixierpunkt. 0 . rechts blasenförmige $A b$ lösung der ganzen oberen Hälfte den Sehnerven und die Netzhautmitte überhängend. Links myopischer Habitus, sonst normal. 25. VII. 11. Die Ablösung senkt sich und ist fast total. $S$ R. = Finger in nächster Nähe. 4. VIII. 11. Nach 3 wöchentlicher ganz vergeblicher friedlicher Behandlung Panktion mit Druekverband. 5. VIII. 11. Obwobl die Hornhaut noch starke streifige Drucktrübungen zeigt, gibt Patient beim Verbandwechsel an, wesentlich besser zu sehen. 7. VIII. 11. Ablösung völlig verschwunden. 12. VIII. 11. Netzhaut liegt überall an. 15. IX. 11. $S$ R. $-4 D$ $\bigcirc-2 D \mathrm{cyl}=5 / 20^{*}$ Gesichtsfeld normal. 0 . keine Spur von Ablösung. 6 Wochen nach der Operation geheilt entlassen, 21. X. 11. Alles gut. 11. XI. 11. Seit 4 Tagen wieder Sehstörung. Schwerer Rückfall nach 14 wöchentlicher Heilung. 18. XI. 11. Wiederaufnahme. $S \mathrm{R}$. = Finger in $3 \mathrm{~m} .0$. Ablösung der ganzen oberen und eines Teils der unteren Hälfte. 28. XI. 11. Nach Senkung der Ablösung heute II. Punktion mit Druckverband. 1. XII. 11. Ablösung wieder ganz beseitigt. 9. I. 12. Die Ablösung ist nieht wiedergekehrt. $S=5 / 35$. Gesichtsfeld normal. Geheilt entlassen. 7. VI, 13. $5 \mathrm{R} .-5 \mathrm{D}={ }^{5} \mathrm{~T}_{20}$. Gesichtfeld normal. 0 . Netzhaut liegt überall an. Also Heilung seit $11 / 2$ Jahren. 
Bei diesem Patienten ist der Erfolg der operativen Behandlung wieder überraschend gut. Fast totale Ablösung bei Myopie, auf die nichtoperative Massnahmen ganz ohne Einfluss sind, wird nach der ersten Operation sofort und vollkommen beseitigt. 8 Wochen nach der Entlassung und 14 Wochen nach der Operation tritt ein Rückfall auf, der bald wieder fast zur alten Ausdehnung der Amotio führt. Die zweite Operation erzielt wiederum sofortige und vollständige Wiederanlegung und Wiederkehr der früheren Funktion. Die Heilung kann heute noch nach $11_{2}$ Jahren konstatiert werden.

Fall 28. Techniker K, 28 Jahre. Derselbe Patient wie Fall 10. Im Mai 1910 Erkrankung des linken Auges an Amotio. Da diese beschränkt bleibt, sogenannte friedliche Behandlung. Im Frühjahr 1912 Verschlimmerung. Anfnahme 27. IV. 12. Ablösung fast der ganzen unteren Hälfte bis zum Sehnerven bei freibleibendem Zentrum. $S=5{ }_{7}$. Gf. Besehränkung von oben bis $40^{\circ}$. 29. IV. 12. Punktion mit Druekverband. 30.IV.12. Amotio vollständig versehwunden. 1. VI. 12. Amotio bleibt fort. $S \mathrm{~L}=5 / 7$. Gesichtsfeld normal. 7. VI. 13. Geheilt nach 13 Monaten.

Auch dieses Auge, indem die Ablösung 2 Jahre bestand, ist durch die Operation, und zwar nur durch eine einmalige zur Heilung geführt. Da jetzt mehr als $1 \mathrm{Jahr}$ seit der Operation verflossen ist, ohne dass ein Rückfall eingetreten, so besteht auch hier Aussicht auf Dauerheilung.

Fall 29. Kaufmann E., 28 Jahre, Myopia magna mit Amblyopie reehts. 20. V. 11. Schlag mit Schirmgriff aufs rechte Auge. 6 Tage später Sehstörung. $S$ R. $-14 D=5 / 60$, L. $-10 D=5 / 7$. Gesichtsfeld hochgradig beschränkt bis auf einen unteren inneren Quadranten. 0 . ausgedehnte blasige Ablösung der oberen und eines Teils der unteren Hälfte. Unter konservativer Behandlung Senkung der Ablösung. 15. VI. 11. Punktion mit Druckverband. 25. VI. 11 nur partielle Besserung, flache Ablösung unten bleibt bestehen. $S$ R. $-14 D=5_{30}$ bei annähernd normalem Gesichtsfeld. Wiederholung der Operation wird verweigert. 14. IX.12. $S$ R. $-6 D=\left.1\right|_{15}$. Gf. Beschränkung von oben bis $10^{\circ}$. 0 . erhabene Ablösung der unteren Hälfte bis nahe an den Sehnerven, die obere Hälfte liegt an.

Die Behandlung war erfolglos. Vielleicht hätte eine Wiederholung der Operation Besserung erzielt.

Fall 30. Frl. Z., 16 Jahre, Myopia excess. April 1911 Myopieoperation rechts anderswo, 11 Discissionen und 2 Lanzenextraktionen. Anfänglich Sehschärfe gut, seit Ende Juni Sehstörung. 3. VII. 11 allseitige Amotio. $S=$ Handbewegungen in sehr kleinem Gesichtsfeld. 9. VIII. 11 Punktion mit Druckverband. 12. VIII. 11. Amotio besteht noch. 23. VIII. 11. Ungebessert entlassen. 
Ein ganz verzweifelter Fall mit absolut schlechter Prognose, indem die Operation nur auf Wunsch der Angehörigen als ein letzter Versuch gemacht wurde.

Fall 31. Näherin V., 20 Jahre. Anfang März 1912 ziemlich plötzliches Auftreten von Sehstörung links. Anfnahme 23. III. 12. $S$ R. $=5_{5}$, L. $=5 / 50^{\circ}$ Gf. Beschränkung von oben bis $30^{\circ}$. 0. Vorgeschrittene Netzhautablösung in der unteren äusseren Hälfte. 19. IV. 12. Unter Bettruhe usw. Fortschreiten der Ablösung. Deshalb Punktion mit Druekverband. 27. IV. 12. Koine Spur von Ablösung mehr. Sehsehärfe besser. 17. V. 12. $S \mathrm{~L}$. $+2 D={ }^{5}{ }_{15}$. Gesichtsfeld normal. 0 . in der Peripherie besteht wieder zarte Áblösung. 21. V. 12. Die Ablösung hat sich mehr ansgedehnt, deshalb II. Punktion mit Druckverband. 23. V. 12. Ablösung fort. 8. VI. 12. Netzhaut überall anliegend. Sehschärfe gut. 21. VI. 12. In der oberen inneren Peripherie wieder zarte Ablösung sichtbar. $S \mathrm{~L}+1,5 \mathrm{D}$ $=\left.{ }^{5}\right|_{15}$. Gesiehtsfeld ohne gröbere Einschränkung. 24. VI. 12. Auf Wunsch entlassen. 22. VII. 12. Die Ablösung ist nieht nur umsehrieben geblieben, sondern erscheint sogar kleiner geworden. Sehschärfe $={ }^{5} / 15$.

Die operative Behandlung hat in diesem Fall, wenn auch keine Heilung, so doch subjektiv und objektiv erhebliche Besserung erzielt, die auch nach Aufnahme der gewohnten Beschäftigung von Dauer blieb.

Fall 32. Fran W., 73 Jahre. Früher gut gesehen. Vor 2 Jahren Erblindung rechts durch Netzhautablösung, seit 2. XI. 12 auch Sehstörung links. Anfahahme 9. XI. 12. Stat. R. Cataracta compl. und Sekundärglaukom. Links änsserlich normal. $S \mathrm{R} .=\frac{1}{\infty}, \mathrm{L}=5 / 15$. Gesichtsfeld hochgradig von oben und unten bis auf $10^{\circ}$ eingeengt. $O$. links fast totale $\mathrm{Ab}$ lösung besonders nach oben, nur temporal und zentral findet sich eine kleine Partie anliegend. In den folgenden Wochen unter der üblichen nicht operativen Behandlong sank die zentrale Sehschärfe rapide, da auch das Zentrum ergriffen wurde. 25. XI. 12. Sehschärfe herabgesetzt bis auf Erkennen von Fingern in $1 \frac{1}{2} \mathrm{~m}$. 2. XII. 12. Punktion mit Druckverband. 3. XII. 12. Keine Spur von Ablösung mehr zu sehen. 7. XII, 12. Nasal besteht wieder flache Ablösung. 18. XII. 12. Flache, aber ausgedehnte Ablösung wieder da. 21. XII. 12 entlassen. $S=$ Finger in $1 / 1 / 4 \mathrm{~m}$.

Das hohe Alter der Patientin, die totale Ablösung der Netzhaut, der Ausgang der Frkrankung auf dem andern Auge musste zu einer denkbar ungünstigen Prognose führen. Die Operation war nur ein erfolgloser Versuch, die sichere Erblindung abzuwehren.

Fall 33. Handlungsgehilfe L., 26 Jahre. Kurzsichtig seit Kindheit. 1897 Erblindung des linken Auges an Netzhautablösung. Am 9. XII. 12 Auftreten von Sehstörung rechts. In Brasilien, wo er sich aufhielt, mit Pilocarpin behandelt. Da der Zustand sich versehlimmerte, fuhr er in seine Heimat, suchte Dr. Mühsams Klinik auf, der den Patient uns am 25. II. 13 überwies. Stat. L. Cataracta compl. bei Phthisis bulbi. R. Äusserlich normal. 
$S$ R. $-12 D=\left.{ }^{5}\right|_{50^{*}}$ Gf. Beschränkung von oben bis $30^{\circ}$. 0 . ausgedehnte Ablösung der ganzen unteren und eines Teils der oberen Hälfte. 10. III. 13. Punktion mit Druckverband. 13. III. 13. Netzhaut liegt überall an. 13. IV. 13. Die Netzhaut bleibt anliegend. Sehschärfe besser. 14. V. Geheilt entlassèn, mit $S-14 D=5 / 20$ und normalem Gf. 30. V. Netzhaut liegt an.

Da fast 12 Wochen nach der Operation verflossen, so ist es wohl angängig, den Fall als einen erfolgreichen, wenn auch noch nicht als gebeilten zu führen. Wie aber auch sein weiterer Verlauf sich gestalten mag, so illustriert er immer die günstige Wirkung der Methode in sinnfälliger Weise; denn es handelte sich um eine weit vorgeschrittene Ablösung, die sich unter der bisherigen Behandlung regelmässig verschlimmert hatte.

Die statistische Berechnung eines Materials, wie des vorliegenden, bietet Schwierigkeiten, and ihr Wert ist nur ein bedingter. Die Fälle sind in anatomischer wie klinischer Hinsicht allzu verschiedenartig; wir haben frische, ältere und ganz veraltete Ablösungen, Fälle mit Emmetropie, leichter, mittlerer und hochgradiger Myopie, Fälle, in denen die Ablösung spontan entstanden and solche, in denen Traumen, Kontusionen und perforierende Verletzungen mit Glaskörperverlust oder Myopieoperationen vorausgegangen sind. Auch sind die Beobachtungszeiten bei einem Teil der Fälle ungenügend. Eine Statistik der operativ behandelten Fälle von Netzháutablösung ist daher nicht $z u$ vergleichen mit einer Statistik typischer Operationen wie z. B. der Kataraktextraktion, bei der die Chancen für die Heilung im grossen und ganzen gleichartige sind.

Meine Statistik hätte ungleich günstiger ausfallen können, wenn ich in der Auswahl der zu operierenden Fälle wählerischer gewesen wäre, wenn ich nur die Erfolg versprechenden Fälle operiert und in den mehr oder weniger aussichtslosen Fällen auf die Operation verzichtet hätte. Sie wurde aber in den letzten Jahren in fast allen Fällen gemacht, die unter der sogenannten friedlichen Behandlung nicht zu bessern waren, einmal, um die Methode möglichst zu erproben, dann, weil manchmal auch in verzweifelten Fällen noch überraschende Wirkungen zu verzeichnen waren. Anderseits ist es wahrscheinlich, dass in diesem oder jenem Falle noch Frfolge hätten erzielt werden können, wenn nicht aus äusseren Gründen die Wiederholung der Operation hätte unterbleiben müissen. Aufzugeben ist meines Erachtens erst ein Fall, wenn 2 Punktionen erfolglos gewesen sind.

Will man versuchen, die Fälle nach dem Resultat der Behandlung zu ordnen, so kann man sie in 4 Gruppen unterbringen. 
I. Fälle, in denen die Operation erfolglos oder so gut wie erfolglos war: Es sind 14 an der Zahl, und zwar Fall 1, 7, 8, 12, 15, $16,17,18,19,24,26,29,30,32$. Bei sämtlichen Fällen dieser Gruppe war von vornherein die Prognose schlecht zu stellen; in 3 Fällen bestand die Ablösung schon über $1 \mathrm{Jahr}, 6 \mathrm{mal}$ betraf sie excessiv myopische Augen, 4 mal waren Traumen, 1 mal mit Glaskörperverlust voransgegangen, $1 \mathrm{mal}$ eine Kataraktextraktion in der Kindheit und 1 mal eine Myopieoperation mit ungewöhnlich zahlreichen Eingriffen. 10 mal war die Ablösung schon eine totale oder fast totale, als sie zur Operation kam.

II. Fälle, in denen die Wiederanlegung nicht erreicht wurde, die aber in gebessertem Zustande entlassen worden sind.

Als solche sind 6 Fälle zu nennen, nämlich Fall 4, 5, 9, 23, 25, 31. In 3 von diesen Fällen bestand hohe Myopie. Die Zeit zwischen Beginn der Ablösung und Operation betrug 6 Wochen bis 4 Monate. Die Besserung war in 4 Fällen nur eine beschränkte, in 2 aber eine erhebliche, insofern als nur Spuren von Ablösung noch nachweisbar blieben. Da aber sämtliche Fälle früher oder später aus den Augen verloren wurden, so lässt sich leider nicht sagen, ob die Besserung auch in den letzteren Fällen von Dauer war.

III. Fälle, in denen die Operation zur Wiederanlegung der Netzhaut geführt hat, und in denen $\mathrm{n}$ ach länger als 3 Monaten ein Rückfall aufgetreten ist.

Hierher gehören die Fälle 6,13 und 14. Wenn sie in klinischer Hinsicht schliesslich auch Misserfolge sind, so zeigen sie doch den günstigen Effekt der Operation und rechtfertigen meines Erachtens diese Sonderung. In allen dreien lag excessive Myopie vor. Im Falle 6 bestand die Ablösung, die allmählich total geworden war, schon 5 Monate, als die Punktion die völlige Wiederanlegung bewirkte. 4 Monate blieb das Auge geheilt bei guter Sehschärfe; dann kam der Rückfall. Eine 2. Punktion erzielte noch einmal für 2 Monate die Wiederanlegung. Der neue Rückfall war dann nicht mehr zu bekämpfen. Im Fall 13 bestand die Ablösung 8 Wochen, als operiert wurde. Die völlige Wiederanlegung der Netzhaut konnte noch nach $3^{1 / 2}$ Monaten festgestellt werden. Im Fall 14 schritt man 6 Wochen nach Beginn der Sehstörung zur Operation. Hier blieb $3 y_{2}$ Jahre das Auge gesund und gut sehend, dann erst wurde es von dem Rückfall heimgesucht. Der Fall war schon als ein Fall von Dauerheilung betrachtet. Während im Fall 6 das Endresultat ganz schlecht ist, so ist der Zustand im Fall 13 und 14 ein derartigex, 
dass die Patienten das erkrankte Auge gebrauchen und so wenig durch ihr Leiden gestört sind, dass sie sich zu der wiederholt vorgeschlagenen Wiederholung der Operation bisher nicht haben entschliessen können.

IV. Fälle, in denen bisher kein Rückfall beobachtet worden ist.

In diese Gruppe können wir 10 Fälle bringen, und zwar Fall 2, $3,10,11,20,21,22,27,28,33$. Es sind 9 Männer und 1 Mädchen im Alter von 20 bis 48 Jahren. 2 mal bestand excessive, 5 mal mittlere, bzw. geringgradige Myopie und 1 mal Emmetropie; in einem Fall war die Ablösung in einem 3 Jahre zuvor wegen Myopie operierten Auge entstanden. Die Ablösung bestand zur Zeit der Operation in 3 Fällen seit 4 Wochen, in 4 Fällen seit $2-3$ Monaten, und in 2 Fällen ungefähr seit einem Jahre. 5 mal erzielte schon die erste, 4 mal erst die zweite Operation Wiederanlegung ron Dauer. Bis auf 1 Fall (11), in dem der jahrelange Bestand der Ablösung zu Atrophie der Netzhaut geführt hatte, war in sämtlichen Fällen eine erhebliche Besserung der Funktion in der Mehrzahl bis zu der früheren Sehschärfe festzustellen. Die Beobachtungszeit der Heilung beträgt 2 mal nur 3-4 Wochen, 2 mal 3-4 Monate, 1 mal $1 \mathrm{Jahr}, 3 \mathrm{mal}$ $11_{2} \mathrm{Jahr}, 1 \mathrm{mal} 4 \mathrm{Jahre}$ und 1 mal 5 Jahre.

Die Fälle 20 and 21 , die schon nach wenigen Wochen auf eigenen Wunseh aus der Klinik entlassen und nicht wieder gesehen wurden, können natürlich nicht als Fälle von Heilung geführt werden, wenn auch anzunehmen ist, dass die Patienten sich im Fall eines Rückfalls wieder vorgestellt hätten. Immerhin dienen sie als Beispiele für die Zweckmässigkeit der Behandlungsmethode.

Birch-Hirschfeld sagt, dass wir die Bezeichnung „Heilung“ erst anwenden dürfen, wenn nach mehrjähriger Beobachtung kein Rezidiv eingetreten ist. Mein Fall 14 der III. Gruppe, der $\left.3{ }_{2}\right|_{2}$ Jahre gesund blieb und dann wieder erkrankte, lehrt, dass man nicht einmal dazu berechtigt ist. Ich möchte daher gar nicht von "Heilung“, sondern nur von "Aussicht auf Dauerheilung“ sprechen.

Bei einer so beschränkten Zahl von Fällen, die ron rornherein prognostisch ganz verschieden zu beurteilen waren, hat es wenig Sinn, Prozentzahlen zu bringen, und es ist nicht angängig, Vergleiche anzustellen mit Statistiken, wie sie Deutschmann bringt, die sich auf ein fast $10 \mathrm{mal}$ so grosses Material gründen ${ }^{1}$. Auch Birch-Hirsch-

1) Deutschmann, Zur Kenntnis der Netzhautablösung und ihrer Behand lung. Festschr. f. Leber, v. Graefe's Arch. f. Ophth. Bd. LXXIV. S. 206. 1910. 
feld hat darauf verzichtet. Ich begnïge mich damit, festzustellen, dass in 10 von 33 Fällen, unter denen sich eine grosse $Z$ ahl fast hoffnungsloser Fälle befand, Aussicht auf Dauerheilung besteht, und dass in 6 von diesen die Wiederanlegung länger als $1 \mathrm{Jahr}$ hindurch beobachtet worden ist. Es sind Fälle, bei denen sämtlich eine mehr oder weniger lange Zeit fortgeführte sogenannte friedliche Behandlung ganz erfolglos gewesen ist, Fälle, die keine Neigung zur Spontanheilung zeigten, die der Erblindung verfallen waren.

Eine Schädigung habe ich niemals durch den Druckverband nach der Punktion gesehen. Kontraindiziert erscheint er mir nur in den Fällen höchstgradiger Kurzsichtigkeit mit sehr verdünnter Sklera und stark prominenten Bulbi, ferner bei brïchigen Gefässen und Neigungen zu Blutungen. Selbstverständlich halte ich immer jeden operativen Eingriff für einen Kunstfehler, wenn die Ablösung nur flach und umschrieben ist und keine Neigung zeigt, sich auszudehnen.

$\mathrm{Ob}$ es angebracht ist, in frischen Fällen mit ausgedehnter und fortschreitender Ablösung die Patienten mit Rückenlage, Schwitzen, subconjunctivalen Kochsalzeinspritzungen usw. za quälen, ist diskutabel. Ich bin jetzt mehr geneigt, die Fälle, in denen wenig Wahrscheinlichkeit für eine Spontananlegung der abgelösten Netzhaut besteht, sitzen und auch etwas umhergehen zu lassen, damit die Senkung der Amotio schneller von statten geht, und die Operation zu machen, sobald die Senkung erfolgt ist. Die jetzt notwendige Bettruhe wird dann williger und besser ertragen. Auf die Kochsalzeinspritzungen unter die Bindehaut habe ich fast ganz verzichtet, einmal, weil ich nie einen sicheren Nutzen davon gesehen habe, weder bei Ablösung, noch bei Netzhautblutungen, Glaskörpertrübungen oder dergl.; dann, weil die auch bei aseptischen Vorgehen nicht ganz zu rermeidenden Reizungen und zuweilen auftretenden Bindehautabscesschen mich öfters gezwungen haben, die Punktion hinauszuschieben. Die Chancen für eine Heilung sind um so besser, je früher die Operation geschieht.

Es muss zugegeben werden, dass diese kombinierte Methode der Punktion und des Druckverbandes die anatomischen Verbältnisse, die für die Genese der Ablösung bedeutungsvoll sind, nur zum Teil berücksichtigt. Eine Sprengung oder Dehnung der G]askörperstränge, bzw. der elastischen präretinalen Membranen wird dadurch nicht erreicht werden, in beschränktem Masse auch nur eine Dehnung der verkürzten Netzhaut. Ich halte es demnach für unwahrscheinlich, dass 
Die Kombination von Punktion und Druckverband zur Behandlung usw. 359

es damit gelingen wird, Fälle zur Heilung zu führen, in denen die Veränderungen im Glaskörper und in der Netzhaut sehr ausgedehnte sind. Ich gebe zu, dass in der Theorie die Deutschmannschen Operationen und die Birch-Hirschfeldschen Glaskörperinjektionen rationeller sind, aber, ob sie es in der Praxis sind, ob durch sie Fülle mit vorgeschrittenen Glaskörper- und Netzhautreränderungen geheilt werden können, ist ebenfalls wenig wahrscheinlich.

Der Hauptrorzug der kombinierten Methode der Punktion und des Druckverbandes ist, dass sie absolut ungefährlich und ohne Risiko ist.

Bei den Glaskörperinjektionen ist und bleibt der wunde Punkt die Möglichkeit des Auftretens einer Trübung des Glaskörpers und einer unkontrollierbaren entzündlichen Reaktion. Diese mag geringer sein, wenn statt Kaninchen- oder Kalbsglaskörper arteigene Stoffe benutzt werden, aber vermieden wird sie damit nicht. Indifferenter ist sicher die Injektion von isotonischer Kochsalzlösung. Birch-Hirschfeld beruft sich bei der Empfehlung seiner Glaskörperinjektionen ausser auf seine eigenen Versuche am Kaninchen auf die erfolgreichen Versuche von Löwensteïn und Samuels ${ }^{1}$ ), sowie die Arbeiten von Elschnig ${ }^{2}$ ), die sich mit dem Ersatz des Glaskörpers durch Kochsalzlösung befassen. Tatsächlich hat Birch-Hirschfeld in den 6 Fällen, in denen er nur Kochsalzlösung zur Injektion benutzte, keine Reaktion erlebt; die Erfolge waren aber auch unbefriedigend; nur 1 Fall wurde erheblich, 2 nur wenig und 3 gar nicht gebessert. Das entspricht der Anschauung Deutschmanns, dass die Kochsalzlösung zu schnell resorbiert wird, um eine Wirkung von einiger Dauer ausüben zu können. Deutschmann gab daher die Einspritzung von Kochsalzlösung zugunsten der von Tierglaskörper wieder auf.

In 6 Fällen, bei denen von Birch-Hirschfeld subretinales Fluidum allein oder mit Kochsalzlösung verdünnt in den Glaskörper gespritzt wurde, trat aber eine mehr oder weniger heftige Reaktion ein, die unter dem Bilde einer Iridocyclitis mit pericornealer Injektion, Trübung des Kammerwassers und des Glaskörpers, Hypopyon, hinteren Synechien usw. verlief. Wenn auch diese Reaktion nur vorübergehend war und in 5 Fällen sogar noch zu einem guten Endresultat geführt hat, so bleibt sie doch immer unliebsam und besorgniserregend, und ich glaube kaum, dass Birch-Hirschfeld heute schon seine

1) Löwenstein u. Samuels, Über Glasleörperersatz. v. Graefe's Arch. f. Ophth. Bd. LXXX. S. 500. 1912.

2) Elschnig, Über Glaskörperersatz. v. Graefe's Arch. f.Ophth. Bd. LXXX. S. 5141912 . 
Methode in einem Falle anwenden wird, in dem, wie z. B. in meinem. Fall 33, das eine Auge bereits erblindet, und das andere an Ablösung erkrankt, aber noch gebrauchsfähig ist. Zurzeit sind die Erfahrungen noch nicht derartige, um die eindringliche Warnung Uhthoffs vor allen Glaskörperinjektionen als unberechtigt hinzustellen und den eingebürgerten Respekt von dem unantastbaren Glaskörper zu beseitigen. Birch-Hirschfeld selbst stellt ja auch seine Versuche noch als Vorversuche hin und sein Verfahren als verbesserungsfähig. Vielleicht werden weitere Erfahrungen unsere Anschauungen vom „noli me tangere" des Glaskörpers ändern. Vorläufig aber möchte ich der Methode der Punktion mit nachfolgendem Druckverband vor den Glaskörperinjektionen den Vorzug geben, wenigstens in Fällen, in denen noch viel $\mathrm{zu}$ verlieren ist.

Ich bin weit davon entfernt, mit diesen Mitteilungen übertriebene Hoffnungen erwecken zu wollen, ich will nur Anregung geben, ein Verfahren zu erproben, das nichts weiter ist als die Kombination zweier seit Albr. v. Graefe's Zeiten geübten Methoden, die jedenfalls ganz unschädlich ist. Ich brauche daher nicht, wie es BirchHirschfeld vorsichtigerweise vorlüufig tut, Bedenken zu tragen, das Verfahren zur allgemeinen Anwendung dem Praktiker zu empfehlen. In meinem in der Berliner ophthalmologischen Gesellschaft über dieses Thema gehaltenen Vortrage sprach ich die Vermutung aus, dass wahrscheinlich hier und da dieses naheliegendo Verfahren schon versucht worden ist, wenn auch die Literatur dariber nichts bringt, zamal Uhthoff die Kombination der Punktion mit subconjunctivalen Kochsalzlösungen empfohlen hat. Ich hatte die Genugtuung, in der Diskussion zu erfahren, dass bereits vor einigen Jahren in der Klinik von Prof: Froehlich einzelne Fälle auf diese Weise mit bestem Erfolg behandelt und ähnliche Erfahrungen gemacht worden sind wie bei uns. Dass diese Methode einfacher ist als alle andern gegen die Netzhautablösung empfohlenen operativen Massnahmen, liegt auf der Hand; auch besteht kein Hinderungsgrund, im Falle eines Misserfolges später die Heilung noch durch Vornahme anderer Eingriffe zu versuchen. 